PAPER

Langevin dynamics with energy dissipation on periodic lattice structures modeling as an equivalent two-body method for atom-surface interactions

To cite this article: Dandan Zeng et al 2019 Mater. Res. Express 6125507

View the article online for updates and enhancements.

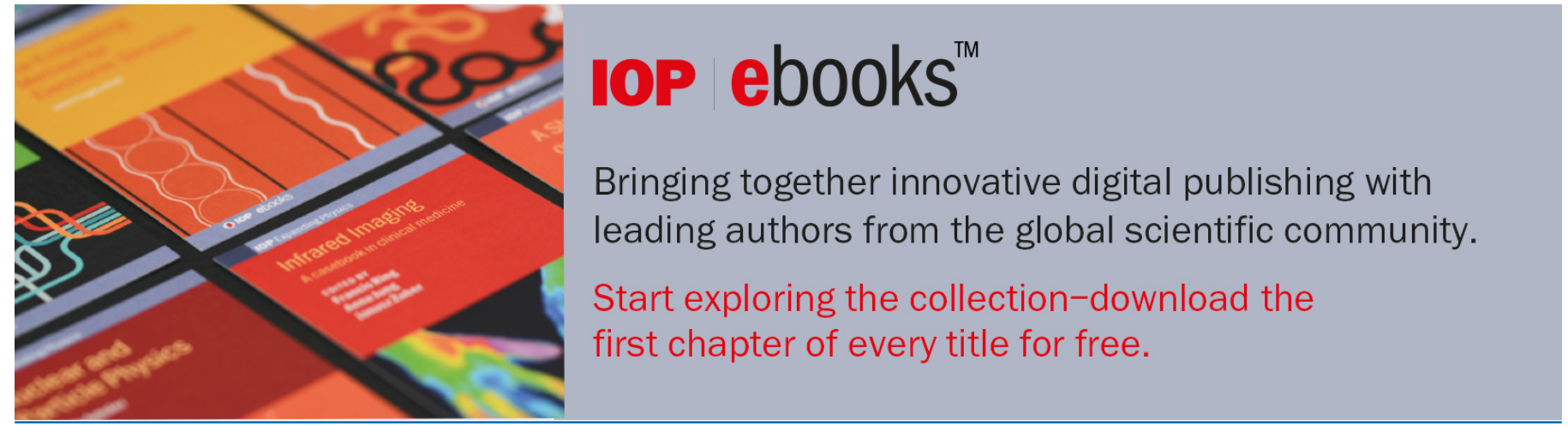

This content was downloaded from IP address 159.226 .231 .70 on 10/04/2020 at 04:00 
PAPER

CrossMark

\title{
Langevin dynamics with energy dissipation on periodic lattice structures modeling as an equivalent two-body method for atom- surface interactions
}

PEVISED 20 October 2019 ACCEPTED FOR PUBLICATION 6 November 2019

PUBLISHED 15 November 2019

\author{
Dandan Zeng $^{1,2}$, Chong Liu ${ }^{1,3,4}\left(\mathbb{D}\right.$, Jianzheng Jiang ${ }^{1,3}$ and Jing Fan ${ }^{1,3,4}$ \\ 1 State Key Laboratory of High Temperature Gas Dynamics, Institute of Mechanics, Chinese Academy of Sciences, Beijing 100190, People's \\ Republic of China \\ 2 Academy of opto-electronics, Chinese Academy of Sciences, Beijing 100094, People's Republic of China \\ 3 School of Engineering Science, University of Chinese Academy of Sciences, Beijing 100049, People's Republic of China \\ 4 Authors to whom any correspondence should be addressed. \\ E-mail: chongliu@imech.ac.cn and jfan@imech.ac.cn
}

Keywords: atom-surface interactions, trapping probability, scattering angle, residence time, dissipating parameters, energy accommodation coefficients

\begin{abstract}
In this work, we propose a classical equivalent two-body (ETB) model that can capture more detailed dynamic features arising from energy dissipation and atom oscillations, by introducing the Langevin equation of a harmonic oscillator. The trapping probability, scattering angle and the residence time of Ar interacting with Pt (111) and W (110) surfaces predicted by the ETB model agree well with the measured experimental data or molecular dynamics simulations. Moreover, the ETB model is also used to study the influence of oscillating and dissipating properties on the thermal accommodation coefficients and rainbow scattering of gas atoms colliding with the surface. It is found that the dependence of energy accommodation coefficients and rainbow scattering on the oscillating and dissipating parameters shows nonlinear behaviors, and the associated mechanisms are disclosed. ETB model further provides the possibility to explore the physics beyond the existing two-body models for a better description of energy transferring during atom surface interactions.
\end{abstract}

\section{Introduction}

The atomic dynamics on surface has been paid special attention due to the crucial role in many scientific and engineering applications. For instance, in vacuum science and technology the pumping down speed is mostly determined by the sticking probability and residence time of the gas atoms interacting with the inner surface of the vacuum system [1-3]. In surface science, the diffraction patterns and the rainbow effects of the scattered atoms has proven to be a useful method for analyzing the surface structure and interaction potential [4-8]. Moreover, the physical and chemical dynamics of the atoms on surface are also relevant with pathways for heterogeneous catalysis, corrosion and crystal growth [9-15]. The energy and momentum accommodations of the gas atoms after colliding could affect the nonequilibrium transport processes in space, plasma, detonation and microchannel [16-18], which have drawn growing interest in atom-surface interactions. Despite the interesting physics and practical applications, the microscopic mechanisms are either unpredictable by the available theories or very difficult to be addressed by experiment due to the lack of measurement with sufficient accuracy, and therefore tremendous efforts have been devoted to understanding the physics of atom-surface interactions.

Simplified classical models provide an efficient way to analyze the physics of atom-surface interactions, even though the quantum model could provide more realistic physical systems than classical model and some features associated with quantum effects cannot be captured by the classical model based on empirical potential functions [19-22]. Actually, in the classical limit of gas surface interactions, large incident energies and high surface temperatures could suppress the quantum effects [23-25], and then classical models could be employed 
to investigate the gas surface interactions. Some underlying mechanisms governing the non-equilibrium phenomena have been revealed by classical models [6,26-28]. Compared with the all-atom molecular dynamics simulations, simplified classical models are much more flexible and have advantages in exploring how different factors, such as surface corrugation, thermal vibration of the solid atoms, thermal conductivity of the solid, etc, influence the atom-surface interactions independently. Although the simplified models may reduce accuracy, the atomic dynamics under different surface conditions can be more quickly evaluated by simplified models than that by all-atoms simulations, this is crucial important for some practical applications. Hence the classical models still play an important role in investigations of atom-surface interactions [16, 29-32].

The complex multibody interactions have been reduced to two-body interactions between gas atom and simplified surface model since 1960s. The first attempt to achieve this is hard cube model [33, 34], in which solid surface is modeled by a hard cube with flat surface. The hard cube model shed light on the effects of temperature and the mass ratio of incident atoms to solid atoms on the scattering angle distribution and energy accommodation coefficient. However, the sticking probability of atoms cannot be predicted by the hard cube model. To circumvent this limitation, the hard cube model has then been extended to the soft cube model [35], which takes the attractive interaction into consideration and then can be used to analyze the influence of potential depth on sticking probability qualitatively. Analogous to the hard cube model, the smoothness assumption in soft cube model ignores the contributions of real surface roughness, which may play an important role in rainbow scattering [36-38], and affect the relative importance of tangential and normal incidence energy for sticking probability. To this end, the washboard model has been proposed to study the corrugation effect $[39,40]$. In washboard model, the corrugated surface is constructed with many continuously distributed hard or soft cubes, and the surface roughness could be modified by changing the cube distributions. Another way of describing the corrugated surface is to view it as a corrugation potential of atom-surface interaction [29, 41-44]. With these approaches, the dynamics of atom-surface interaction could be more accurately extracted from classical models.

As far as we know there have been a great deal of works using the simplified classical models investigating how the mass ratio, incident energy, surface temperature, potential well depth and surface corrugation affect atom-surface interactions. These parameters coupled with the energy dissipation processes make atom-surface interaction more complicated, e.g., the energy dissipation of solid atoms may change the vibrational induced corrugations on surface and consequently affect the atom-surface interactions [45, 46]. In fact, the energy dissipation of the surface atoms has been considered by some classical models, such as the generalized Langevin equation (GLE) method [47] and the generalized Langevin oscillator (GLO) model of the surface [48]. In GLE model, solid substrate is constructed with regularly arranged atoms, the motions of the solid atoms indirectly interacting with incident atoms are described by the GLE equation, while motions of the solid atoms directly interacting with the incident atoms are governed by the spring equation, however, this method works like simplified MD method, and lacks the advantages of two-body model in studying the underlying mechanism of atom-surface interaction. The GLO model is a two-body model, the model describes the surface as a smooth generalized Langevin oscillator, in which the corrugation effect of the surface is not taken into consideration. To further explore how the oscillating and dissipating properties influence atom-surface interactions, we propose an equivalent two body (ETB) model with the intention of providing a precise description of atom-surface interaction through including the energy dissipation terms. The ETB model is constructed and introduced in section 2, and is validated by applying it to three classical atom-surface interaction problems in section 3 . After that, we use the model to analyze the influence of dissipating and oscillating properties of the solid atom on thermal accommodation coefficients in section 4 . At the end, a brief conclusion and discussion about the applicability and extension of the model is presented.

\section{Framework of equivalent two body model}

\subsection{Basic ideas}

The reduced classical two-body models have been traditionally used as an efficient tool to describe nonequilibrium atom-surface interactions. The energy dissipation and solid atom oscillation may have essential influences on the atom-surface interactions. When a gas atom moves toward the solid surface, the potential energy converts to the kinetic energy of the gas atom, and finally some of them converts to the kinetic energy of the solid atoms after collision. The locally heated solid atoms could subsequently transfer energy to the surrounding solid atoms and then keep thermal equilibrium with them. The energy transfer processes are mainly achieved through the gas-solid interactions and solid-solid interactions. To capture the fundamental physics on the surface which is coupled with energy transfer, we propose an equivalent two-body (ETB) system, which incorporates energy dissipation terms to model the atom-surface interactions. In our ETB model, the solid atoms which are directly colliding with gas atoms are defined as the primary solid atom, while the other 
atoms on the surface are classified as subordinate atoms. Physically, the primary solid atom can be reckoned as the solid atom which influences the gas motion the most.

Let $\boldsymbol{r}_{\mathrm{g}}=\left(x_{\mathrm{g}}, y_{\mathrm{g}}, z_{\mathrm{g}}\right), \boldsymbol{r}_{\mathrm{s}}=\left(x_{\mathrm{s}}, y_{\mathrm{s}}, z_{\mathrm{s}}\right)$ be the coordinates of the gas atom and the primary atom respectively, $\boldsymbol{r}=(x, y, z)=\left(x_{\mathrm{g}}-x_{\mathrm{s}}, y_{\mathrm{g}}-y_{\mathrm{s}}, z_{\mathrm{g}}-z_{\mathrm{s}}\right)$ is the relative position between them, $z$ direction is perpendicular to the solid surface. The interaction potential between gas and primary atoms is represented by $\phi(x, y, z)$, which is estimated from the interaction potential between gas atom and solid surface. The force exerted on the solid atom can be described by generalized Langevin equation (GLE) [49, 50]. The simplest version of the GLE, the Brownian equation of a harmonic oscillator is used to describe the motion of the solid atoms [51], and the validity of the equation will be verified in the next subsection. Based on the above analysis, the motion of the two bodies can be described by

$$
\begin{gathered}
\ddot{\boldsymbol{r}}_{\mathrm{g}}(t)=-\frac{\nabla_{\mathrm{r}_{\mathrm{g}}} \phi}{m_{\mathrm{g}}} \\
\ddot{\boldsymbol{r}}_{\mathrm{s}}(t)=-\frac{1}{m_{\mathrm{s}}} \boldsymbol{k} \cdot \boldsymbol{r}_{\mathrm{s}}-\boldsymbol{\zeta} \cdot \dot{\boldsymbol{r}}_{\mathrm{s}}+\boldsymbol{A}(t)-\frac{\nabla_{\mathrm{r}_{\mathrm{s}}} \phi}{m_{\mathrm{s}}}
\end{gathered}
$$

where $\boldsymbol{k}$ and $\boldsymbol{\zeta}$ are diagonal matrices, the diagonal components of $\boldsymbol{k}$ and $\boldsymbol{\zeta}$ are written as $\boldsymbol{k}_{\boldsymbol{i}}, \zeta_{\boldsymbol{i}}(i=x, y, z)$, which represent the spring constant and dissipation coefficient in $x, y, z$ directions. $A(t)$ is gaussian fluctuation force, its component $A_{i}(\mathrm{t})$ equals to $\sqrt{2 \zeta_{i} k_{\mathrm{B}} T / m_{s}} \eta$, where $\eta$ is a uniformly distributed random number in the range of $(0,1)$. According to the fluctuation-dissipation theorem, $A_{i}(t)$ and $\zeta_{i}$ satisfy the following relationship,

$$
\left\langle A_{i}(t) A_{i}(\tau)\right\rangle=2 \zeta_{i} k_{\mathrm{B}} T / m_{s} \delta(t-\tau), i=x, y, z
$$

where $k_{\mathrm{B}}$ is the Boltzmann constant, $T$ is the temperature of the solid, $\delta$ is the Dirac function.

The square root of $k_{i} / m_{\mathrm{s}}$ determines oscillating frequency of the solid atom, and the relaxation speed of the solid atom to the equilibrium state could be affected by $\zeta_{i}$, since large $\zeta_{i}$ could enhance the energy dissipation of the primary atoms. The values of $k_{i}$ and $\zeta_{i}$ involve both microscopic and macroscopic parameters, which include the interaction strength, the separation of solid atoms and the temperature of the solid. Through experiments or MD simulations, these parameters of specific gas-surface system can be determined for the analysis of ETB model.

\subsection{Oscillating and dissipating parameters}

To obtain the oscillation and dissipation parameters, the Brownian equation of oscillator for the motion of solid atoms is validated as follows. Taking the motion in $x$ direction as an example, by eliminating the force exerted on the primary atom by gas atom, equation $(2.2)$ reduces to

$$
\ddot{x}_{s}(t)=-\frac{1}{m_{\mathrm{s}}} k_{x} x_{\mathrm{s}}-\boldsymbol{\zeta}_{x} \cdot \dot{x}_{\mathrm{s}}+A_{x}(t)
$$

The corresponding velocity auto-correlation function $C(t)=\left\langle\dot{x}_{\mathrm{s}}(\tau) \dot{x}_{\mathrm{s}}(t+\tau)\right\rangle$ equals to [51]

$$
C(t)=C(0) e^{-\zeta_{x} t / 2}\left(\cos w t-\frac{\zeta_{x}}{2 w} \sin w t\right), w=\sqrt{k_{x} / m_{s}-\zeta_{x}^{2} / 4}
$$

we compare the velocity auto-correlation function obtained by equation (2.5) with the MD simulation results to check the validity of equation (2.4) for the motion of solid atoms. The MD simulations are performed using the Large-scale Atomic/Molecular Massively Parallel Simulator (LAMMPS) [52] developed at the Sandia National Laboratories.

As representatives of low energy surfaces with face centered cubic (fcc) and body centered cubic (bcc) crystals, the motions of atoms on $\mathrm{Pt}$ (111) and W (110) surface are studied respectively. In MD simulations, the embedded-atom (EAM) potentials are used to calculate the interaction between solid atoms [53, 54]. After the solid reaches thermal steady state, velocity auto-correlation functions of the solid atoms in the topmost layer are sampled and averaged. Figures 1 and 2 illustrate $C(t)$ for $\mathrm{Pt}(111)$ at $273 \mathrm{~K}$ and W (110) at $373 \mathrm{~K}$ as a function of time, where the symbols show the MD results and the solid lines are the fitted results based on equation (2.5). It is seen that the fitted results based on equation (2.5) agree well with the MD results, which indicates that the thermal motion of solid atoms can be well described by Brownian motion of harmonic oscillator.

We then evaluate the values of $k_{i} / m_{\mathrm{s}}$ and $\zeta_{i}(i=x, y, z)$ by fitting the velocity auto-correlation functions obtained by MD simulations to equation (2.5). Table 1 lists the values of $k_{i} / m_{\mathrm{s}}$ and $\zeta_{i}(i=x, y, z)$ of atoms on $\mathrm{W}(110)$ and $\mathrm{Pt}(111)$ surfaces at different temperatures. It is shown that $k_{i}$ is almost invariant as the temperature changes within the range considered in this work, this is because the interaction strength and the separation of solid atoms do not change too much with temperature. For $\zeta_{i}$, it is found that $\zeta_{x}$ and $\zeta_{y}$ are insensitive to temperature due to the strong confinement in directions parallel to the surface; Nevertheless, in the direction perpendicular to the surface $\zeta_{\mathrm{z}}$ varies essentially with temperature, the deviation between the maximum $\zeta_{\mathrm{z}}$ with 


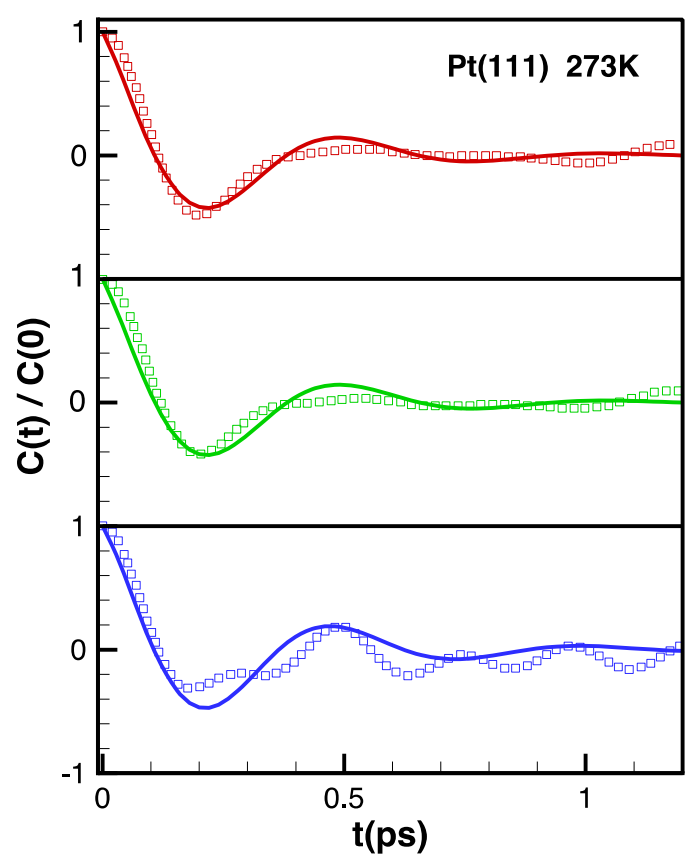

Figure 1. The velocity auto-correlation functions of motion in $x, y, z$ (top, middle, below) directions of $\mathrm{Pt}(111)$ surface atoms at $273 \mathrm{~K}$. Symbol: results of MD simulation, solid line: equation (2.5) with $\boldsymbol{k}_{\boldsymbol{i}}$ and $\boldsymbol{\zeta}_{\boldsymbol{i}}$ listed in table 1.

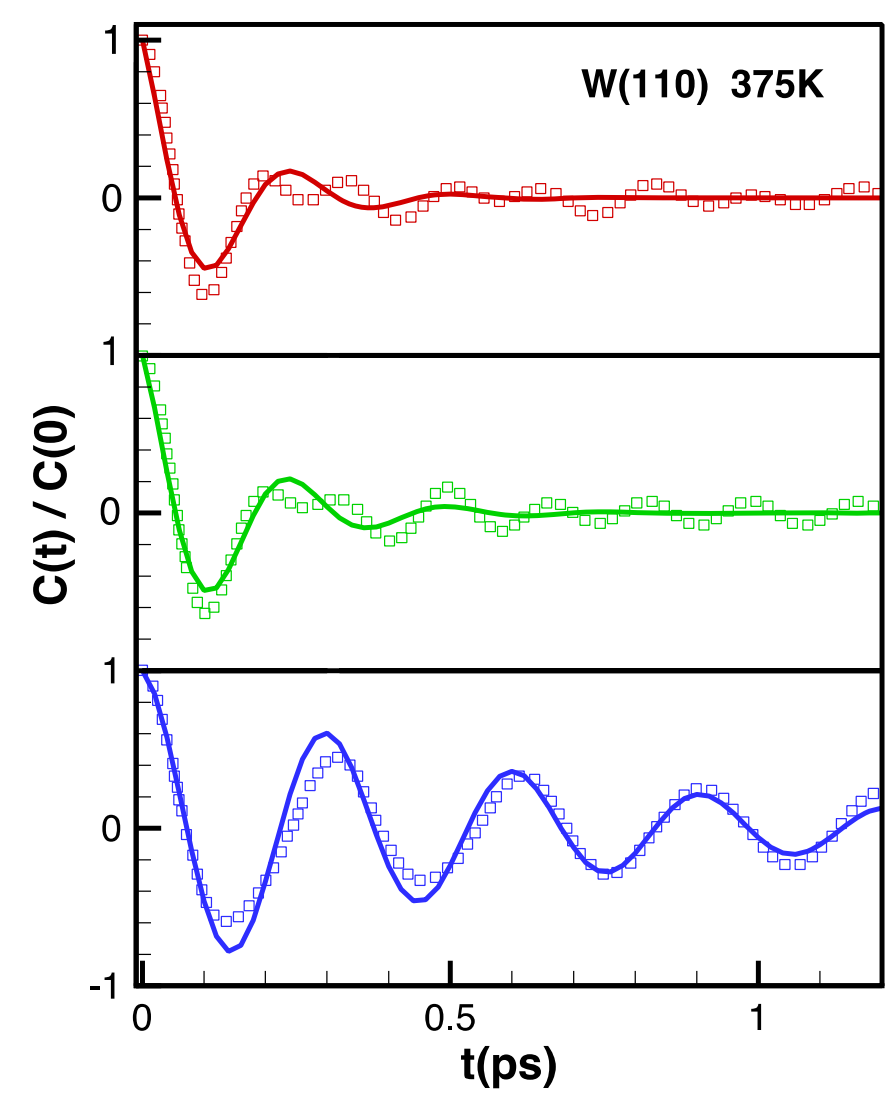

Figure 2. The velocity auto-correlation functions of motion in $x, y, z$ (top, middle, below) directions of W(110) surface atoms at $375 \mathrm{~K}$. Symbol: results of MD simulation, solid line: equation (2.5) with $\boldsymbol{k}_{\boldsymbol{i}}$ and $\boldsymbol{\zeta}_{\boldsymbol{i}}$ listed in table 1.

respect to the minimum $\zeta_{\mathrm{z}}$ is no larger than $21 \%$ and $15 \%$ for $\mathrm{Pt}(111)$ and $\mathrm{W}(110)$ surface respectively. The less sensitivity of the dissipating and oscillating parameters to temperature may facilitate the application of the ETB model under different temperatures. 
Table 1. $k_{i} / m_{s}\left(\mathrm{ps}^{-2}\right)$ and $\zeta_{\boldsymbol{i}}\left(\mathrm{ps}^{-1}\right)$ of $\mathrm{Pt}(111)$ and $\mathrm{W}(110)$ surface atoms at different temperatures.

\begin{tabular}{lccccccc}
\hline Surface & $T(\mathrm{~K})$ & $\zeta_{x}$ & $k_{x} / m_{\mathrm{s}}$ & $\zeta_{y}$ & $k_{y} / m_{\mathrm{s}}$ & $\zeta_{z}$ & $k_{z} / m_{\mathrm{s}}$ \\
\hline $\mathrm{W}(110)$ & 375 & 14.9 & 625 & 12.9 & 640 & 3.39 & 430 \\
& 575 & 15.1 & 640 & 12.5 & 630 & 3.52 & 421 \\
& 775 & 15.1 & 632 & 12.2 & 614 & 3.90 & 409 \\
$\operatorname{Pt}(111)$ & 80 & 8.19 & 149 & 8.19 & 149 & 8.56 & 158 \\
& 190 & 7.95 & 152 & 7.93 & 149 & 7.30 & 162 \\
& 273 & 7.93 & 148 & 7.89 & 147 & 7.05 & 160 \\
\hline
\end{tabular}

\subsection{Interaction potential of gas atom and solid surface}

The interaction between a pair of neutral atoms or molecules, $i$ and $j$, can be described by the Lennard-Jones (LJ) potential function, $\varphi_{i j}\left(r_{i j}\right)=4 \varepsilon\left[\left(\sigma / r_{i j}\right)^{12}-\left(\sigma / r_{i j}\right)^{6}\right]$, where $r_{i j}$ is the intermolecular distance, $\varepsilon$ is the molecular binding energy, and $\sigma$ is the collision diameter. The LJ parameters have been extensively investigated and their values for many popular species are available in the literature $[55,56]$. If the interacting atoms are of different species, the potential parameters can be calculated by the Lorentz-Berthelot mixing rule. For the interaction between a gas atom $i$ and a surface in our proposed ETB model, the potential can be assumed to be the sum of interactions between the atom and each constituent atoms of the surface,

$$
\phi_{i}(r)=\sum_{j=1}^{N} \varphi_{i j}\left(r_{\mathrm{g}-\mathrm{s}, \mathrm{j}}\right)
$$

where $r_{\mathrm{g}-\mathrm{s}, j}$ is the distance between the gas atom and the $j$ th solid atom of the surface, $N$ is the total number of the atoms on the surface. Equation (2.6) is a simple approach to obtain the atom-object interaction potentials and the idea has also been widely used to determine the gas-particle potentials and the gas-carbon nanotube (CNT) potentials [57-59]. For gas-surface interaction, the soft cube model [35] and the washboard model [39, 40] assume the solid atoms are continuously and uniformly distributed on the surface, which may lead to some artificial features. To this end, the physical discrete configuration of surface atoms is taken into account in this work. For a given surface with cubic crystal structure, the lattice constant along three different directions are equal and we refer to $b$. The surface roughness arising from lattice structures can be characterized by $\sigma / b$, where $\sigma$ is the interaction distance between the gas atom and the solid atom. Here, we employ equation (2.6) to numerically calculate $\phi(x, y, z)$ and plot the dependences of $\phi$ on $z$ at hollow and top site for different $\sigma / b$ in figures 3 and 4 . To consider the lattice effect, $\sigma / b$ is varied to cover a wide range of surface conditions through modifying $\sigma$. It is seen from figures 3 and 4 that the difference of potential between the hollow site and the top site is small for large $\sigma / b$, because large $\sigma / b$ could reduce the surface roughness.

The equilibrium positions of solid atoms are periodically allocated on an idealized crystalline surface, e.g. fcc or bcc, and therefore the potential $\phi$ on time average follows periodic distribution according to its lattice structure. For the convenience of applying analytical potential function in equations (2.1) and (2.2), we fit the exact interaction potential from equation (2.6) to its analytical formulation. Different periodical analytical formulations have been developed for the surfaces with different lattice structures, equations (2.7) and (2.8) are proposed for fcc (111) and bcc (110) surfaces respectively:

$$
\begin{aligned}
& \phi=A \varepsilon_{\mathrm{g}-\mathrm{s}}\left[\left(\frac{\sigma_{\mathrm{m}}^{2}}{z^{2}+S}\right)^{6}\left(1+\frac{S-S_{0}}{b^{2}}\right)^{5}-\left(\frac{\sigma_{\mathrm{m}}^{2}}{z^{2}+S_{0}}\right)^{2}\right], \sigma_{\mathrm{m}}=0.92 \sigma \\
& S(x, y)=\frac{S_{0}}{2}\left(\cos ^{2} \frac{\pi x}{b_{\mathrm{m}}}+\cos ^{2} \frac{\pi y}{b_{\mathrm{m}}}\right), b_{\mathrm{m}}^{2}=\frac{\sqrt{3} b^{2}}{4}, S_{0}=\frac{b^{2}}{8} \\
& \phi=A \varepsilon_{\mathrm{g}-\mathrm{s}}\left[\left(\frac{\sigma_{\mathrm{m}}^{2}}{z^{2}+S}\right)^{6}\left(1+\frac{S-S_{0}}{b^{2}}\right)^{2.5}-\left(\frac{\sigma_{\mathrm{m}}^{2}}{z^{2}+S_{0}}\right)^{2}\right], \sigma_{\mathrm{m}}=0.92 \sigma \\
& S(x, y)=\frac{S_{0}}{2}\left(\cos ^{2} \frac{\pi x}{b_{\mathrm{m}}}+\cos ^{2} \frac{\pi y}{b_{\mathrm{m}}}\right), b_{\mathrm{m}}^{2}=\frac{b^{2}}{\sqrt{2}}, S_{0}=0.225 b^{2}
\end{aligned}
$$

where $A$ is a normalization coefficient making $\phi_{\min }=-\varepsilon_{\mathrm{g}-\mathrm{s}}, \varepsilon_{\mathrm{g}-\mathrm{s}}$ is the binding energy between the gas and the surface. The value of $\varepsilon_{\mathrm{g}-\mathrm{s}}$ can be determined by ab initio calculations, or MD simulations on condition that the interaction of gas atom and solid atom is known. In addition, it can also be measured experimentally based on the thermal effect in desorption [60]. $S(x, y)$ is a periodical function of surface coordinates, and the periodical unit is a square with a $b_{\mathrm{m}}$ side, corresponding to a $1 / b_{\mathrm{m}}^{2}$ area density of atoms, which equals to the virtual area density on surface. For fcc (111) surface, the atomic number density is $4 / \sqrt{3} b^{2}$, which equals to $1 / b_{\mathrm{m}}^{2}$ in equation (2.7), while for bcc (110) surface, the atomic number density is $\sqrt{2} / b^{2}$, which equals to $1 / b_{\mathrm{m}}^{2}$ in 

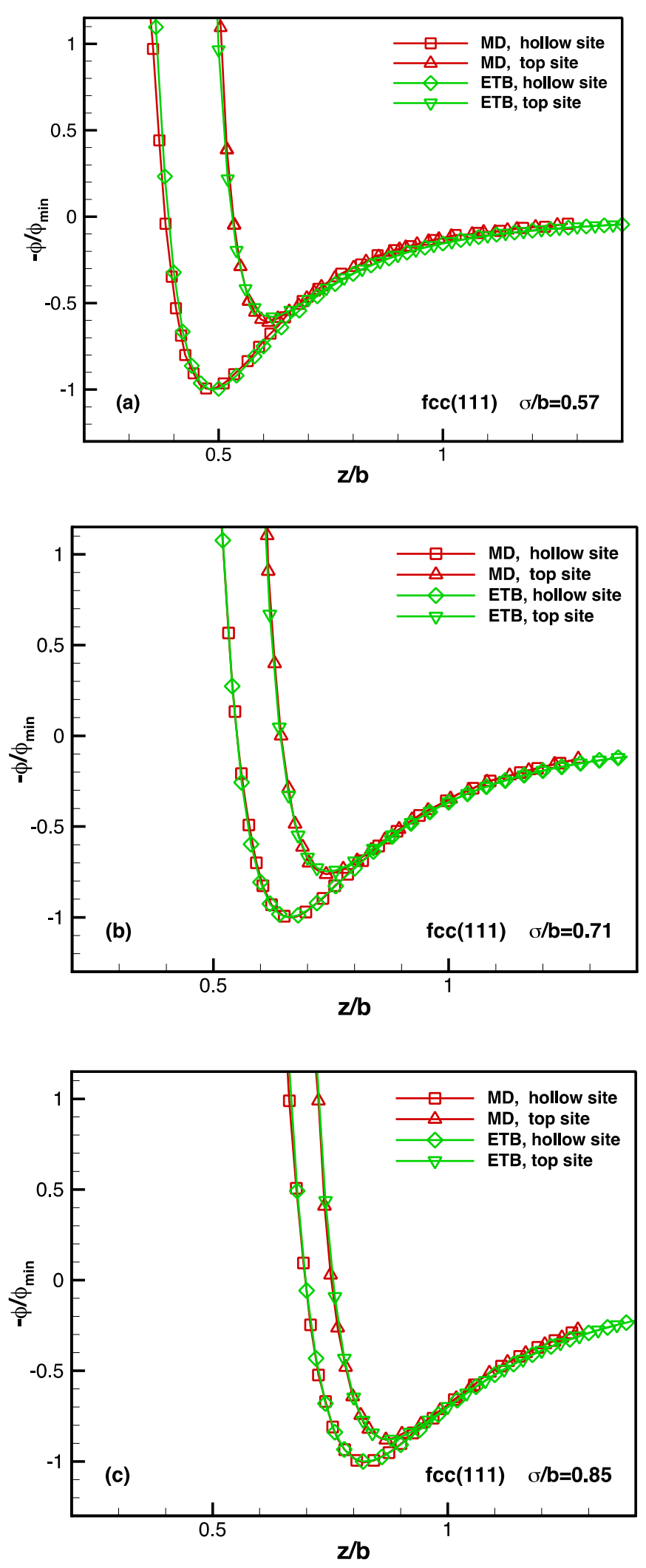

Figure 3. The interaction potential between gas atom and fcc (111) surface. (a) $\sigma / b=0.57$, (b) $\sigma / b=0.71$ and (c) $\sigma / b=0.85$.

equation (2.8). $\sigma_{\mathrm{m}}$ denotes the interaction distance between the gas atom and the surface, $\sigma_{\mathrm{m}}$ is expected to be smaller than the interaction distance between the gas atom and surface atom because the gas atom interacted with more than one atom on the surface. The dependence of $\sigma_{\mathrm{m}}$ on $\sigma$ and that of $S_{0}$ on $b$, together with the potential distribution function are obtained by fitting the gradients of the potential along the normal and tangential direction of the surface to those of the potential obtained by MD simulations for a wide range of $\sigma$ and $b$. 

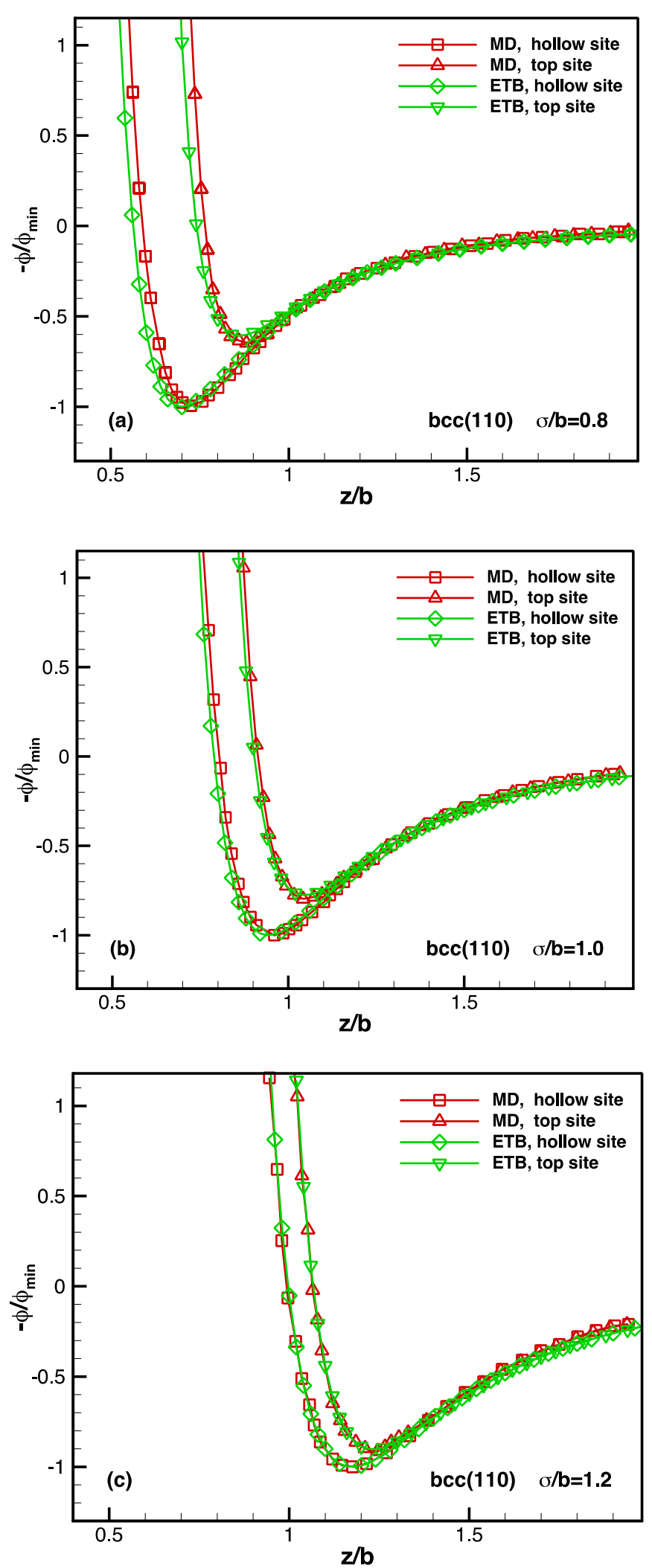

Figure 4. The interaction potential between gas atom and bcc (110) surface. (a) $\boldsymbol{\sigma} / \boldsymbol{b}=0.8$ (b) $\sigma / b=1.0$ and (c) $\boldsymbol{\sigma} / \boldsymbol{b}=1.2$

For both atom-fcc (111) surface and atom-bcc (110) surface interactions, the fitted potentials compare well with the exact potentials under different $\sigma / b$, as illustrated in figures 3 and 4 . The interaction potentials studied above are obtained by assuming that all solid atoms are rigidly fixed at lattice sites. However, solid atoms may deviate from the lattice site with small displacements due to thermal motions, and the vibration of the solid atoms could affect the atom-surface interaction potential. To evaluate the potential deviation, we define the following quantities, 
Table 2. Interaction potential parameters of Ar and Pt(111), W(110) surfaces.

\begin{tabular}{lcc}
\hline Atom-surface & $\sigma(\AA)$ & $\varepsilon_{\mathrm{g}-\mathrm{s}} / k_{\mathrm{B}}(\mathrm{K})$ \\
\hline Ar-Pt(111) & 2.97 & 664 \\
Ar-W(110) & 4.02 & 378 \\
\hline
\end{tabular}

Table 3. The deviation of interaction potential caused by thermal motions of solid atoms at different temperatures.

\begin{tabular}{llcccc}
\hline Atom-surface & $T(\mathrm{~K})$ & 100 & 300 & 500 & 700 \\
\hline \multirow{2}{*}{$\operatorname{Ar}-\operatorname{Pt}(111)$} & $\Delta \phi^{\prime}{ }_{\max }$ & $8.52 \mathrm{E}-02$ & $1.74 \mathrm{E}-01$ & $4.62 \mathrm{E}-01$ & $1.86 \mathrm{E}+00$ \\
& $\left\langle\Delta \phi^{\prime}\right\rangle$ & $2.27 \mathrm{E}-02$ & $4.37 \mathrm{E}-02$ & $6.45 \mathrm{E}-02$ & $1.30 \mathrm{E}-01$ \\
$\operatorname{Ar}-\mathrm{W}(110)$ & $\Delta \phi^{\prime}{ }_{\text {max }}$ & $8.52 \mathrm{E}-02$ & $8.48 \mathrm{E}-02$ & $1.66 \mathrm{E}-01$ & $2.40 \mathrm{E}-01$ \\
& $\left\langle\Delta \phi^{\prime}\right\rangle$ & $1.79 \mathrm{E}-02$ & $2.89 \mathrm{E}-02$ & $4.26 \mathrm{E}-02$ & $5.40 \mathrm{E}-02$ \\
\hline
\end{tabular}

$$
\begin{gathered}
\Delta \phi^{\prime}(x, y, T)=\left|\frac{\phi_{\min }(x, y, T)-\phi_{\min }(x, y, \text { equi })}{\phi_{\min }(x, y, \text { equi })}\right| \\
\Delta \phi_{\text {max }}^{\prime}=\left\langle\Delta \phi^{\prime}(x, y, T)\right\rangle_{x, y, \max } \\
\langle\Delta \phi\rangle=\left\langle\Delta \phi^{\prime} x, y, T\right\rangle_{x, y, \text { ave }}
\end{gathered}
$$

where $\phi_{\min }(x, y$, equi) is the minimum potential along $y$ direction for specific $(x, y)$ when the solid atoms are rigidly located at the equilibrium lattice sites, $\phi_{\min }(x, y, T)$ is the minimum potential when the solid atoms vibrate at temperature $T .\langle\cdot\rangle_{x, y, \max }$ and $\langle\cdot\rangle_{x, y, \text { ave }}$ denote the maximum and averaged value of $\Delta \phi^{\prime}(x, y, T)$ in $(x$, y) plane respectively.

To evaluate the fluctuation of potential caused by thermal motion, we study $\Delta \phi^{\prime}(x, y, T)$ of $\operatorname{Ar}-\operatorname{Pt}(111)$ and $\mathrm{Ar}-\mathrm{W}(110)$ surfaces at different temperatures through MD method. The potential parameters of Ar and solid atoms are listed in table 2. We use the potential parameters given in [61, 62] for Ar-W interactions and [63] for Ar-Pt interactions. The interaction distance of Ar-Pt is arithmetic average of that of Ar-Ar [55] and Pt-Pt [56]. In MD simulations, $\Delta \phi^{\prime}(x, y, T), \Delta \phi^{\prime}$ max and $\left\langle\Delta \phi^{\prime}\right\rangle$ are calculated at an instant after the solid reaches thermal steady state. As shown by the results in table 3 , the deviation caused by thermal motion increases with temperature. Moreover, the deviation for Ar-W (110) is also shown to be smaller than that for $\mathrm{Ar}-\mathrm{Pt}(111)$, because the strong interaction of solid $\mathrm{W}$ atoms corresponds to small amplitude of thermal vibrations.

So far the methodology to obtain the parameters in ETB model has been introduced. In the next section, we apply this ETB model to calculate sticking probability, outgoing angle and residence time in atom-surface interactions.

\section{Application to atom-surface interactions}

\subsection{Sticking probability of Ar colliding with $\mathrm{Pt}(111)$ surface}

Sticking probability of Ar on $\mathrm{Pt}$ (111) surface has been widely studied by experiments and numerical simulations [64-66]. To validate the ETB model, we apply it to calculate trapping probability of Ar on $\mathrm{Pt}(111)$ surface and compare the results with experimental results measured by Mullins et al [66]. In [66], the molecular beams of Ar are incident on $\mathrm{Pt}(111)$ surface with surface temperature of $80 \mathrm{~K}$ and $190 \mathrm{~K}$, the distribution of time of flight of the outgoing atoms under different incident energy and angle is measured, and the trapping probability under different incident energy and angle is obtained by analyzing the distribution of time of flight of the scattered atoms.

The calculations based on ETB model are performed under a range of surface temperatures from $80 \mathrm{~K}$ to $190 \mathrm{~K}$ and a range of the incidence energies $E_{\mathrm{i}}$ from $0.01 \mathrm{eV}$ to $0.8 \mathrm{eV}$ with the incidence angle $\theta_{i}$ varying from $30^{\circ}$ to $60^{\circ}$. Interaction potential between $\operatorname{Ar}-\mathrm{Pt}(111)$ are described by equation (2.7), the potential parameters are listed in table 2 , besides, oscillating and dissipating parameters of the surface listed in table 1 . In our simulations, trapping is defined to occur when the gas atom does not leave the surface within $10 \mathrm{ps}$ after its arriving at the surface. Trapping probability for every case was obtained based on samples of 2000 trajectories, which gives a maximum statistical standard deviation about 0.02 . The results obtained by ETB model are compared with experimental results in figure 5. The dependence of trapping probability on incidence energy and incidence angle satisfies $s \sim E_{\mathrm{i}} \cos ^{\gamma} \theta_{\mathrm{i}}$, and $\gamma$ decreases with increasing $T$. Specifically, when $\gamma=2, s$ is actually a function of normal incidence energy, a decrease in $\gamma$ is associated with the increasing importance of 

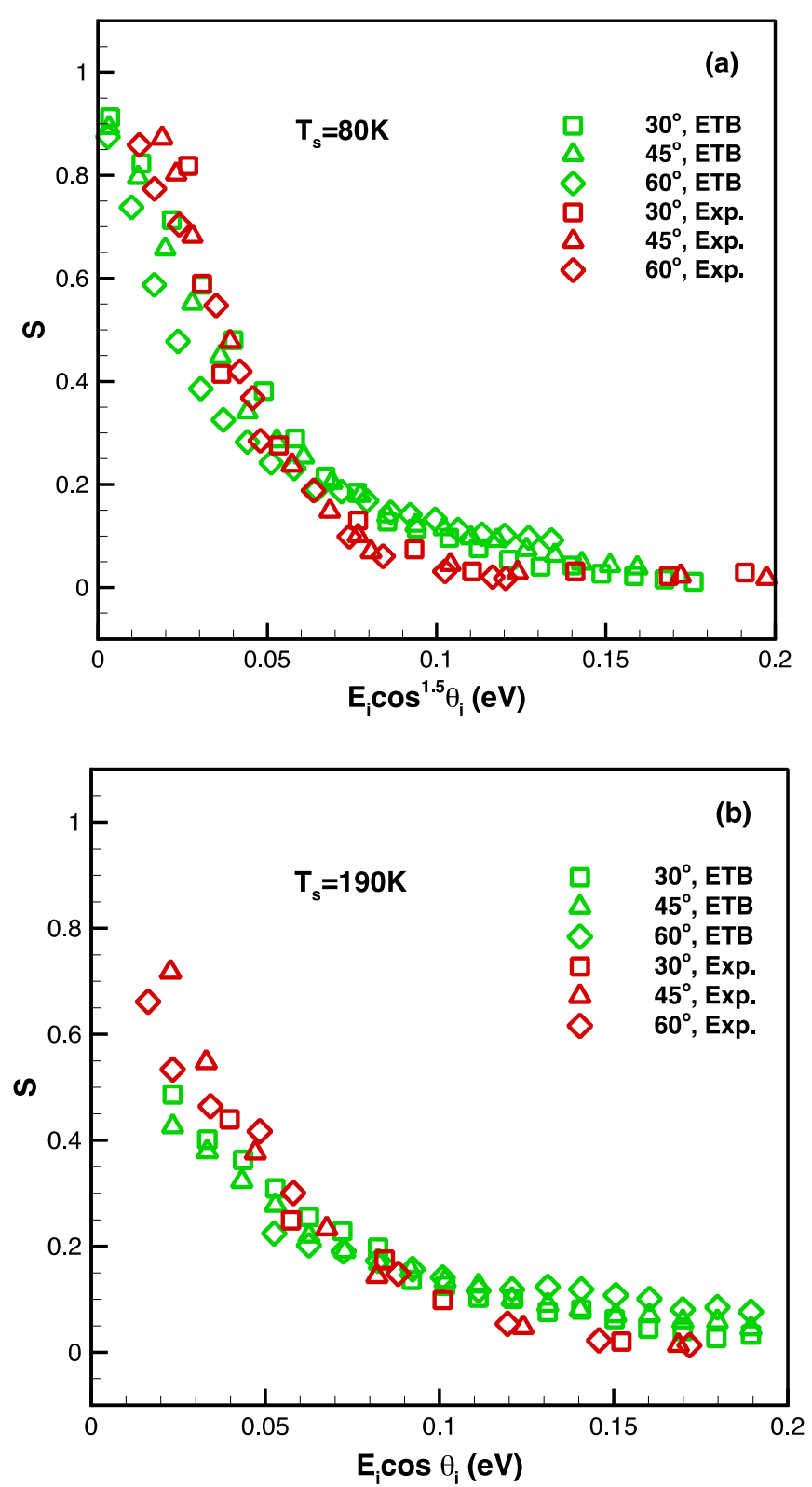

Figure 5. Variation of sticking probability of Ar-Pt (111) collisions with incident energy and incident angle obtained by ETB model and experiments under different surface temperature (a) $T_{\mathrm{s}}=80 \mathrm{~K}$, (b) $T_{\mathrm{s}}=190 \mathrm{~K}$.

tangential energy in reflection. In a whole, trapping probabilities obtained by ETB model agree well with experimental results, the small discrepancies between experimental and ETB results for low incidence energy are possibly caused by the potential deviations induced by thermal motion of solid atoms, which has not been considered in ETB model.

\subsection{Outgoing angle of Ar scattering from $W(110)$ surface}

The scattering of atomic and molecular particles has proven to be a useful method for obtaining a wide variety of information on the structure and dynamics of surfaces [4-8]. Therefore, it is important to predict the outgoing angle of a gas atom scattering from the solid surface [67]. In this section, ETB model is applied to calculate the outgoing angle of Ar scattering from W(110) surfaces.

For the convenience of comparison, the simulation conditions are set to be the same with those of molecular beam experiments reported by Weinberg [68]. In [68], the argon molecules with temperature of $295 \mathrm{~K}$ were incident on W(110) surface with different surface temperatures, and the in-plane intensity of the scattered atoms are measured.

The angle of the incident gas is $45^{\circ}$, and the temperature of solid is changed from $375 \mathrm{~K}$ to $775 \mathrm{~K}$, as shown in figure 6. In ETB simulations, the conditions of the incident atoms and surface are consistent with that of the experiment, and the interaction potential of Ar-W(110) are described by equation (2.8), the potential parameters 

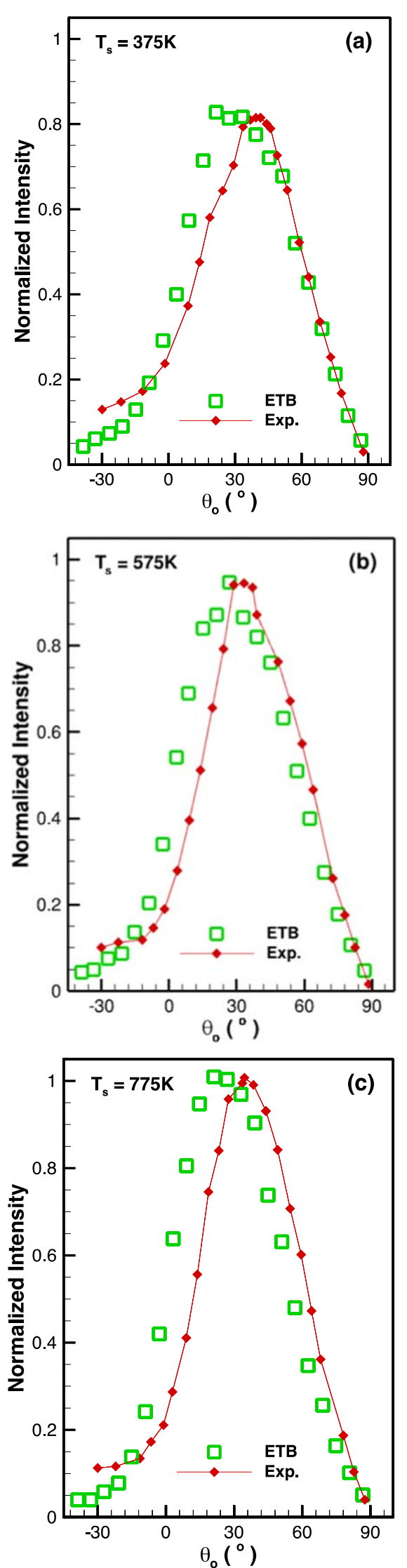

Figure 6. Relative distributions of scattering angle of Ar incident on W (110) surface, the temperature of the incident gas is $295 \mathrm{~K}$, the incidence angle is $45^{\circ}$, for different surface temperatures. (a) $T_{\mathrm{s}}=375 \mathrm{~K}$, (b) $T_{\mathrm{s}}=575 \mathrm{~K}$ and (c) $T_{\mathrm{s}}=775 \mathrm{~K}$. All the distribution functions are normalized by the crest value of $T=775 \mathrm{~K}$. 
Table 4. Residence time of Ar on W(110) surface obtained by MD and ETB method at different temperatures.

\begin{tabular}{lcc}
\hline$T(\mathrm{~K})$ & $\tau_{\mathrm{d}}(\mathrm{ps}, \mathrm{MD})$ & $\tau_{\mathrm{d}}(\mathrm{ps}, \mathrm{ETB})$ \\
\hline 75 & 2067 & 1777 \\
100 & 708 & 844 \\
125 & 431 & 511 \\
\hline
\end{tabular}

are listed in table 2 , and the oscillating and dissipating parameters of the surface listed in table 1 . The motion of a gas atom is numerically integrated up to $100 \mathrm{ps}$ after its arriving on the surface. If the atom does not leave the surface till $100 \mathrm{ps}$, the outgoing velocity of the gas atom satisfies Maxwell distribution, corresponding to a $\cos \theta_{\mathrm{o}} / 2$ distribution function of the outgoing angle $\theta_{\mathrm{o}}$. Through recording the outgoing angle $\theta_{\mathrm{o}}$ of molecules scattered in the principal plane, the distribution functions are obtained. In figures 6(a)-(c), the distribution function for different temperatures predicted by the ETB model are compared with experiment, it is seen that the ETB model predicts the outgoing angle well for all temperatures.

\subsection{Residence time of Ar adsorbed on W(110) surface}

The residence time $\tau_{\mathrm{d}}$ is defined as the expected time needed for an adsorbed gas atom to escape from the surface. Residence time has been seldom studied by previous models, mostly because of the complication to model the continuous bounces of gas atom on the solid surface. In this work, the ETB model is also employed to calculate the residence time for Ar adsorbed on W(110), and the results are compared with MD results.

In both ETB and MD simulations, the gas atoms were initially adsorbed on the surface, and then desorbed at some time triggered by thermal motion. The fraction of molecules remains adsorbed on the surface versus time is obtained from 4000 trajectories. In MD simulations, the 4000 trajectories are calculated in one simulation, i.e., 4000 atoms are initially adsorbed on a surface of $400 \AA \times 400 \AA$ area, and the interactions between gas atoms are ignored. In ETB model, 4000 trajectories are independently calculated.

For rarefied gas atoms adsorbed atoms on an ideal surface, the desorption rate is mainly determined by the binding energy, the surface temperature and coverage rate. In this work, the coverage rate is very small, while the binding energy and the surface temperature is constant, therefore, the desorption rate is proportional to $\Gamma$, Theoretically, the number of gas atoms adsorbed on the surface $\Gamma$ satisfys the following equation

$$
d \Gamma / d t=-\Gamma / \tau_{\mathrm{d}}
$$

and thus $\Gamma=\Gamma_{0} e^{-t / \tau_{\mathrm{d}}}$, with $\Gamma_{0}$ the initial value of $\Gamma$. By fitting the $\ln \Gamma / \Gamma_{0}$ to a linear function of $t$, we obtain $\tau_{\mathrm{d}}$. Table 4 lists $\tau_{\mathrm{d}}$ obtained for different temperatures, and shows that the results obtained by ETB method and MD method compare well with each other, the relative difference is no larger than $20 \%$, while the numerical cost for one trajectory simulation with ETB model is much cheaper than that of MD method.

It is shown that our model could capture more fundamental physics on the surface, including energy transfer between the atom and the surface, energy transfer among solid atoms, and the effect of surface corrugation for atom-surface interaction. The existing two-body models either capture only a part of these processes $[35,39,40,48]$ as discussed in the introduction or incorporate some artificial parameters [42-44]. While all the parameters in our model, including the dissipating and oscillating parameters and the potential parameters are well defined and can be fitted through numerical method or assigned by experimental data. Moreover, it is also worthy to note that the proposed potential function could provide more accurate description of the interaction potential compared with the existing two-body models, such as the hard cube model [33,34], the soft cube model [35], the washboard model [39, 40], the GLO model [48] and the perturbation theory for atom-surface interaction potential [42-44]. Therefore, this model could be employed to investigate the physical properties associated with the energy conversion and dissipation for atom-surface interaction.

\section{Influence of oscillating and dissipating properties on atom-surface interaction}

\subsection{Thermal accommodation coefficients}

The exchange of energy between gas atoms and surface is typically characterized in terms of the energy accommodation coefficient. Extensive efforts have been focused on the influence of gas temperature, solid temperature, potential well depth and mass ratio on thermal accommodation coefficient [16, 30, 35]. However, the influence of oscillating and dissipating properties of solid atoms has seldom been studied. The properties of solid surface determine the energy transfer process and could play an important role in the results of atomsurface interaction. Therefore, it is nontrivial to understand how the dissipating and oscillating parameters 


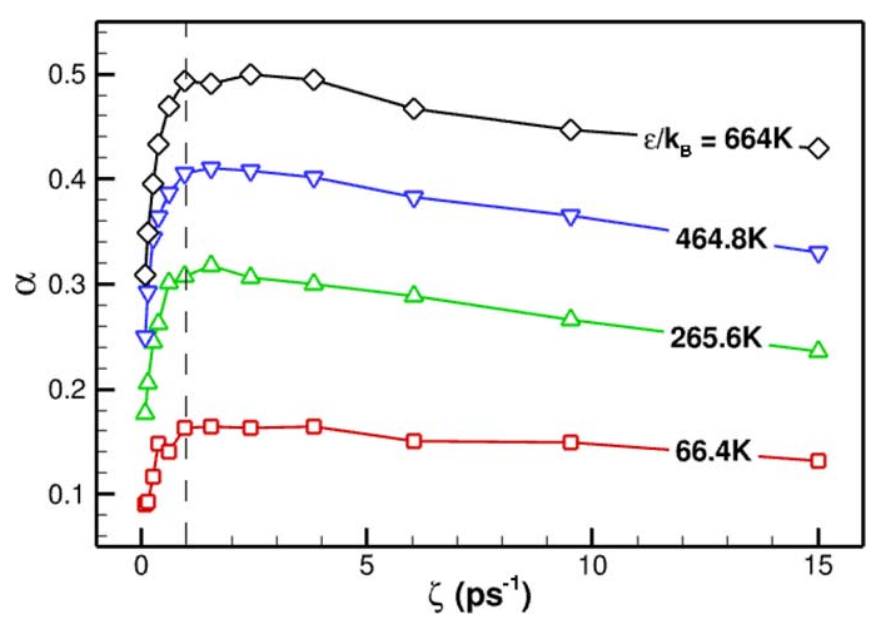

Figure 7. Thermal accommodation coefficients as a function of dissipation coefficients under different $\varepsilon_{\mathrm{g}-s} / k_{\mathrm{B}}$. The atom-surface interaction potential is described by equation (2.7), $\sigma=2.97 \AA$ and $=3.95 \AA, m_{\mathrm{g}}=40 \mathrm{amu}, T_{\mathrm{g}}=300 \mathrm{~K}, m_{\mathrm{s}}=195 \mathrm{amu}$, $k / \mathrm{m}_{\mathrm{s}}=160 \mathrm{ps}^{-2}$.

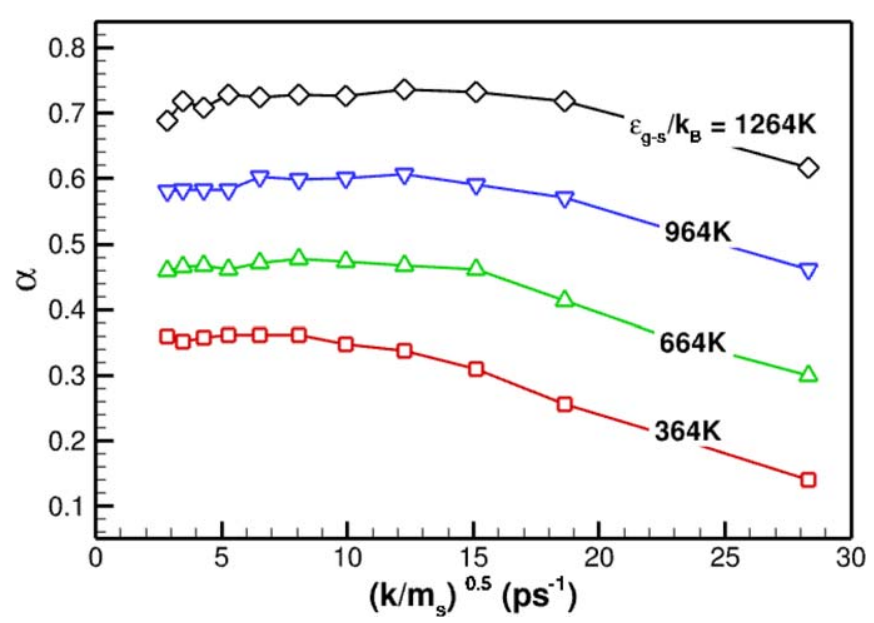

Figure 8. Thermal accommodation coefficients as a function of oscillating coefficients under different $\varepsilon_{\mathrm{g}-\mathrm{s}} / \mathrm{k}_{\mathrm{B}}$. The atom-surface interaction potential is described by equation (2.7), $\sigma=2.97 \AA$ and $b=3.95 \AA . m_{\mathrm{g}}=40 \mathrm{amu}, T_{\mathrm{g}}=300 \mathrm{~K}, m_{\mathrm{s}}=195 \mathrm{amu}$, $\zeta=7 \mathrm{ps}^{-1}$.

influence the thermal accommodation coefficient $\alpha$ which is defined as

$$
\alpha=\frac{T_{\mathrm{o}}-T_{\mathrm{g}}}{T_{\mathrm{s}}-T_{\mathrm{g}}}
$$

where $T_{\mathrm{s}}$ and $T_{\mathrm{g}}$ are temperatures of the solid and incident gas respectively, $T_{\mathrm{o}}$ stands for the temperature of the atoms scattered from the surface. $T_{\mathrm{o}}$ equals to $\left\langle E_{\mathrm{o}}\right\rangle / 2 k_{\mathrm{B}}$, where $\left\langle E_{\mathrm{o}}\right\rangle$ is the average kinetic energy of the scattered atoms.

For atom-surface interaction described by ETB model, the temperature of the scattered atoms $T_{\mathrm{o}}$ depends on the following factors: $m_{\mathrm{g}}, T_{\mathrm{g}}, m_{\mathrm{s}}, T_{\mathrm{s}}, b_{\mathrm{m}}, \boldsymbol{k}, \boldsymbol{\zeta}, \varepsilon_{\mathrm{g}-\mathrm{s}}, \sigma_{\mathrm{m}}$. For the purpose of simplicity, the oscillating and dissipating movements is assumed to be isotropic, and the tensors $\boldsymbol{k}$ and $\boldsymbol{\zeta}$ are reduced to scalars $k$ and $\zeta$ respectively. For all the calculations in this section, the atom-surface interaction potential is described by equation (2.7), where $\sigma=2.97 \AA, b=3.95 \AA, m_{\mathrm{g}}=40 \mathrm{amu}, T_{\mathrm{g}}=300 \mathrm{~K}, m_{\mathrm{s}}=195 \mathrm{amu}, \zeta$ and $k$ is variable. The gas incident on the surface with random azimuthal and incident angles, the temperature of the incident gas equals to $T_{\mathrm{g}}$.

The accommodation coefficients $\alpha$ are plotted as a function of $\zeta$ and $\left(k / m_{\mathrm{s}}\right)^{0.5}$ for different $\varepsilon_{\mathrm{g}-\mathrm{s}} / k_{\mathrm{B}}$ in figures 7 and 8 . It is seen that $\alpha$ increases monotonically with $\varepsilon_{\mathrm{g}-\mathrm{s}}$, because for large $\varepsilon_{\mathrm{g}-\mathrm{s}}$ atoms could bounce more times with high thermal vibration frequency and then facilitate energy dissipation. However, the variation of $\alpha$ with $\zeta$ is more complicated and interesting. For $\zeta<1 \mathrm{ps}^{-1}, \alpha$ increases steeply with $\zeta$, while for $\zeta>1 \mathrm{ps}^{-1}, \alpha$ decreases slowly with $\zeta$. To explain this, we would like to mention that the kinetic and potential 


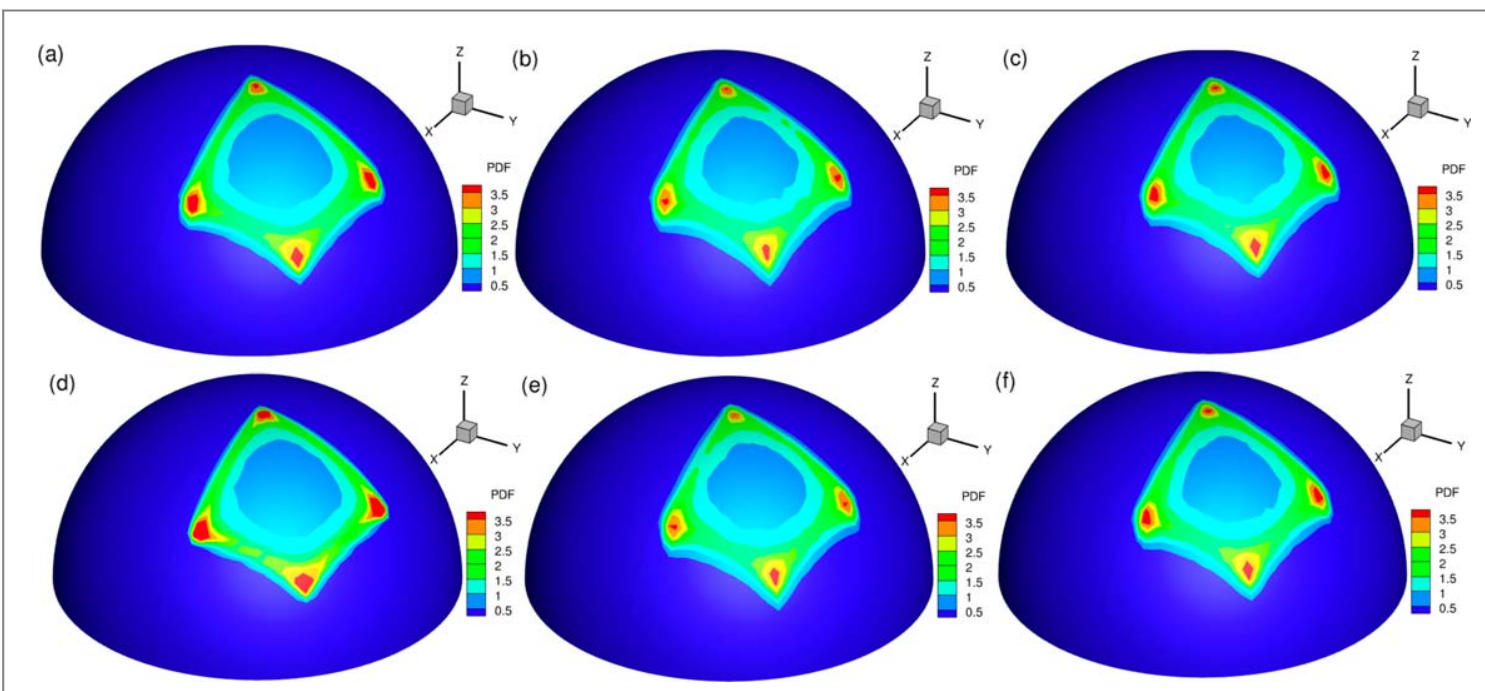

Figure 9. Dependence of angular distribution on dissipating and oscillating properties for an atom scattering from a solid surface. The atom-surface interaction potential is described by equation (2.7) with $\varepsilon_{\mathrm{g}-\mathrm{s}} / k_{\mathrm{B}}=664 \mathrm{~K}, \sigma=2.97 \AA$ and $b=3.95 \AA$. Mass of the incident atom is $40 \mathrm{amu}$, incident energy is $2.4 \mathrm{eV}$, the incident direction is $(\sqrt{2} / 2, \sqrt{2} / 2,-\sqrt{3})$. Mass of the solid atom is $195 \mathrm{amu}, k_{x} / m_{\mathrm{s}}=k_{y} / m_{\mathrm{s}}=150 \mathrm{ps}^{-2}, \zeta_{\mathrm{x}}=\zeta_{\mathrm{y}}=8 \mathrm{ps}^{-1} . \zeta_{\mathrm{z}}=0 \mathrm{ps}^{-1}$ (a), (d), $8 \mathrm{ps}^{-1}$ (b)-(e), $16 \mathrm{ps}^{-1}$ (c), (f), and $k_{\mathrm{z}} / m_{\mathrm{s}}=150 \mathrm{ps}^{-2}(\mathrm{a})-(\mathrm{c}), 290 \mathrm{ps}^{-2}(\mathrm{~d})-(\mathrm{f})$.

energy of incident atom transfers into the kinetic energy of solid atoms at speed of $-\dot{\boldsymbol{r}}_{\mathrm{s}} \cdot \nabla_{r_{\mathrm{s}}} \phi$ during collision, and then the kinetic energy of the solid atom is dissipated at speed of $-\zeta \dot{\boldsymbol{r}}_{\mathrm{s}} \cdot \dot{\boldsymbol{r}}_{\mathrm{s}}$. When $\zeta$ is small, energy cannot be efficiently dissipated in time $t_{\mathrm{b}}$, the dissipation process is the bottleneck for the energy accommodation of the gas atom. Therefore, $\alpha$ increases with $\zeta$. However, as $\zeta$ is increased further, the energy can be efficiently dissipated when $\zeta$ is sufficiently large, and the accelerated (by $-\dot{\boldsymbol{r}}_{\mathrm{s}} \cdot \nabla_{r_{\mathrm{s}}} \phi$ ) solid atom cool down rapidly to $T_{\mathrm{s}}$ with the module of $\dot{\boldsymbol{r}}_{\mathrm{s}}$ being small, and then the energy transfer efficiency $\left(-\dot{\boldsymbol{r}}_{\mathrm{s}} \cdot \nabla_{\mathrm{r}_{\mathrm{s}}} \phi\right)$ could be reduced, which leads to a decrease of $\alpha$, as shown by figure 7 .

The variation of $\alpha$ with elastic coefficient can also be divided into two stages, as shown in figure 8 . In the first stage where $\left(k / m_{\mathrm{s}}\right)^{0.5}$ is small, the thermal accommodation coefficient $\alpha$ is almost independent of $\left(k / m_{\mathrm{s}}\right)^{0.5}$. When $\left(k / m_{\mathrm{s}}\right)^{0.5}$ is large, the thermal accommodation coefficient $\alpha$ decreases with the increase of $\left(k / m_{\mathrm{s}}\right)^{0.5}$. This phenomenon could be explained as follows. When the elastic coefficient is small, solid atoms vibrate with a low frequency, the interaction with the incident atom and the dissipating force dominate over the thermal motion of the solid atoms and the energy exchange processes; when $\left(\mathrm{k} / \mathrm{m}_{\mathrm{s}}\right)^{0.5}$ is large, the elastic force becomes important and an extremely large elasticity coefficient can be viewed as a hard wall surface, which may suppress the energy exchange, therefore thermal accommodation coefficient $\alpha$ decreases.

\subsection{Rainbow scattering}

Angular distribution for scattering of an atom collided with a corrugated surface could have double peaked structure, which is known as rainbow scattering. In this section, the effect of dissipating and oscillating properties on rainbow scattering will be investigated by ETB model, and the influence of dissipation effect on scattering angle will be explored.

To explore the dissipation effect, we fix the other parameters in our calculations. The potential of atomsurface interaction is described by equation (2.7), the value of $\varepsilon_{\mathrm{g}-\mathrm{s}}$ satisfies $\varepsilon_{\mathrm{g}-\mathrm{s}} / k_{\mathrm{B}}=664 \mathrm{~K}$, the interaction distance $\sigma$ is $2.97 \AA$, the lattice constant $b$ is $3.95 \AA$. The mass of the incident atoms is set to be $40 \mathrm{amu}$, the atoms incident on the surface with energy of $2.4 \mathrm{eV}$ along the direction of $(\sqrt{2} / 2, \sqrt{2} / 2,-\sqrt{3})$. The mass of the solid atom is set to be $195 \mathrm{amu}$, the oscillating parameters in $x$ and $y$ directions are characterized by $k_{x} / m_{\mathrm{s}}=k_{y} / m_{\mathrm{s}}=150 \mathrm{ps}^{-2}$, the dissipating parameters $\zeta_{\mathrm{x}}=\zeta_{\mathrm{y}}=8 \mathrm{ps}^{-1}$, the values of $k_{z}$ and $\zeta_{\mathrm{z}}$ are variable and their influences on scattering angle are investigated.

Figure 9 illustrates the distribution of scattering angle under the condition of $\zeta_{\mathrm{z}}=0 \mathrm{ps}^{-1}(\mathrm{a}),(\mathrm{d}), 8 \mathrm{ps}^{-1}(\mathrm{~b})$, (e), $16 \mathrm{ps}^{-1}$ (c), (f), and $k_{\mathrm{z}} / m_{\mathrm{s}}=150 \mathrm{ps}^{-2}$ (a)-(c), $290 \mathrm{ps}^{-2}$ (d)-(f). It is found that the distribution of scattering is symmetrical about the incident plane because the interaction potential is symmetrical about the same plane, besides, rainbow scattering for simulation cases. In practice, the surface structure could be determined by symmetrical properties of distribution of scattering angle by changing the azimuthal angles of the incident gas. To better investigate the influence of dissipating and oscillating parameters on rainbow scattering. Figure 10 displays the probability distribution function (PDF) of the scattering angle in the incident plane. It is seen that the influence of the dissipating and oscillating parameters on the PDF of $\theta_{\mathrm{o}}$ is negligible for $\zeta_{\mathrm{z}} \geqslant 8 \mathrm{ps}^{-1}$. When 


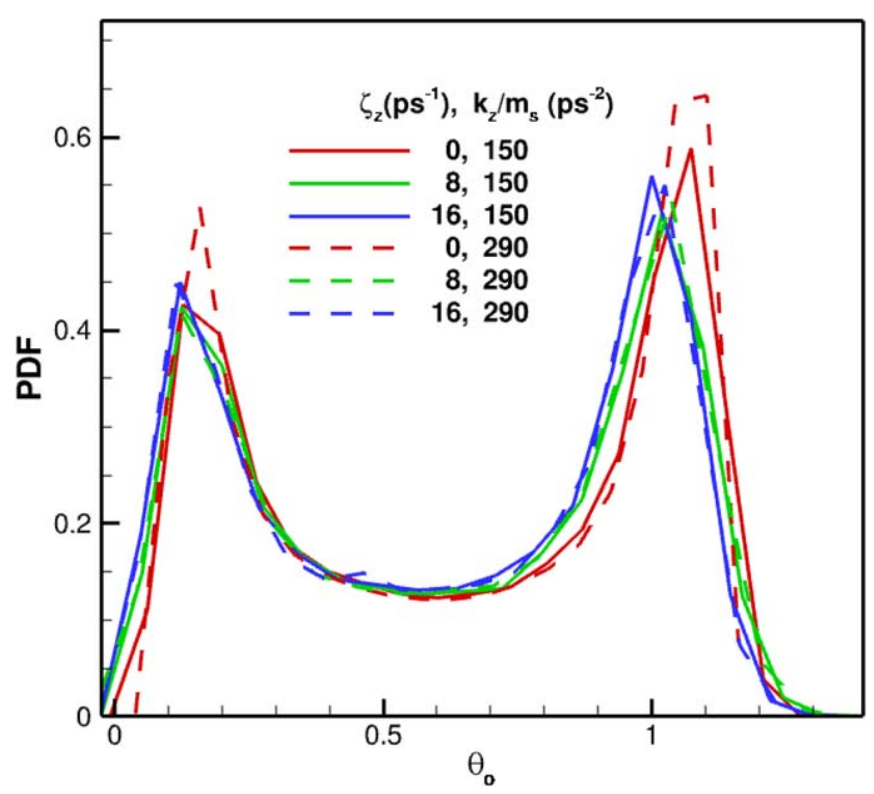

Figure 10. Comparison of in-plane PDF of $\theta_{0}$ shown in figure 9 for different dissipating and oscillating parameters.

$\zeta_{\mathrm{z}}=0 \mathrm{ps}^{-1}$, the PDF of $\theta_{\mathrm{o}}$ is sharper around the two crest for bigger $k_{\mathrm{z}}$ and more rigid surface, because the rigid the surface could enhance the rainbow effect. The above analysis indicates that the rainbow scattering is insensitive to the dissipating and oscillating properties for large $\zeta_{z}$, however when $\zeta_{z}$ is small, the rainbow effect increases with $k_{\mathrm{z}}$ and decreases with $\zeta_{\mathrm{z}}$.

\section{Conclusions and discussions}

In summary, a classical ETB model incorporating energy dissipation is proposed and applied to calculate the sticking probability, outgoing angle and residence time of $\operatorname{Ar}$ on $\mathrm{Pt}(111)$ and $\mathrm{W}(110)$ surfaces. The calculations show that results of the ETB model agree well with that obtained by experiments and MD simulations, which confirms the validity of the ETB model for atom-surface interactions. The ETB model is utilized to investigate the influence of dissipating and oscillating parameters on thermal accommodation coefficients and rainbow scattering. It is found that the dependence of energy accommodation coefficients on $\zeta$ shows nonmonotonic behaviors, which is caused by the competition of energy dissipation and energy transfer efficiency. Moreover, energy accommodation coefficients are almost independent of $\left(k / m_{\mathrm{s}}\right)^{0.5}$ when $\left(k / m_{\mathrm{s}}\right)^{0.5}$ is small. As $\left(k / m_{\mathrm{s}}\right)^{0.5}$ increases, the elastic force becomes dominant, which may suppress the energy exchange and the energy accommodation coefficients drop. For rainbow scattering, the influence of oscillating and dissipating parameters on the distribution of scattering angles is ignorable when dissipating parameter is big. When the dissipating parameter is small, the rainbow effect increases with increasing oscillating parameter and decreasing dissipating parameter. In addition, due to the surface defects and impurities, most practical surfaces are not smooth. The ETB model can be further developed to take these factors into consideration through modifying the potential depth and other parameters variable with surface coordination. The further optimized model could provide an efficient tool to study adsorption, desorption and diffusion problems for gas atoms interacting with heterogeneous surfaces.

\section{Acknowledgments}

This work is supported by the National Natural Science Foundation of China (Grant No. 11302227) as well as by the Youth Innovation Promotion Association CAS (Grant No. 2015014). Funding from the Strategic Priority Research Program of the Chinese Academy of Sciences is also acknowledged.

\section{ORCID iDs}




\section{References}

[1] Dobrozemsky R, Menhart S and Buchtela K 2007 J. Vac. Sci. \& Technol. A 25551

[2] Joly J P, Gaillard F, Peillex E and Romand M 2000 Vacuum 59854

[3] Shiokawa Y and Ichikawa M 1998 J. Vac. Sci. \& Technol. A 161131

[4] Zugarramurdi A, Debiossac M and Lunca-Popa P 2013 Phys. Rev. A 88012904

[5] Schüller A and Winter H 2008 Phys. Rev. L. 100097602

[6] Miret-Artés S and Pollak E 2012 Surf. Sci. Rep. 67161

[7] Hayes W W and Manson J R 2006 Phys. Rev. B 74073413

[8] Shichibe H, Satake Y, Watanabe K, Kinjyo A, Kunihara A, Yamada Y, Sasaki M, Hayes W W and Manson J R 2015 Phys. Rev. B 91 155403

[9] Acharya S R, Shah S I and Rahman T S 2017 Surf. Sci. 66242

[10] Guan J, Huang X, Xu X, Zhang S, Jia X, Zhu X, Wang W and Guo J 2018 Surf. Sci. 669198

[11] Kołaczkiewicz J and Oleksy C 2018 Surf. Sci. 66957

[12] Zhang X, Li B, Liu H X, Zhao G H, Yang Q L, Cheng X M, Wong C H, Zhang Y M and Lim C W J 2019 Appl. Surf. Sci. 465871

[13] Garcia E A, Iglesias-Garcia A, Carrillo S C G M and Romero M A 2018 Appl. Surf.Sci. 452507

[14] Lu J W, Day B S, Fiegland L R, Davis E D, Alexander W A, Troya D and Morris J R 2012 Prog. Surf. Sci. 87221

[15] Liu C, Wang L, Zheng Y, Zeng D, Jiang J and Fan J 2018 J. Appl. Phys. 124235306

[16] Mane T, Bhat P, Yang V and Sundaram D S 2018 Surf. Sci. 677135

[17] Mehta N A and Levin D A 2017 J. Thermophys. Heat Tr. 31757

[18] Giri A and Hopkins P E 2016 J. Chem. Phys. 144084705

[19] Varga G 2002 J. Phys.-Condes. Matter 146081-6107

[20] Balazs E, Varga G and Fustoss L 2001 Surf. Sci. 4821145

[21] Varga G 1999 Surf. Sci. 441472

[22] Varga G and Fustoss L 1991 Surf. Sci. 24323

[23] Hayes W W, Ambaye H and Manson J R 2007 J. Phys.-Condes. Matter 19305007

[24] Al Taleb A, Anemone G, Hayes W W, Manson J R and Farias D 2017 Phys. Rev. $B 95075414$

[25] Hayes W W, Al Taleb A, Anemone G, Manson J R and Farias D 2018 Surf. Sci. 67820

[26] Bortolani V and Levi A C 1986 Riv. Nuovo Cimento 9 1-77

[27] Gerber R B 1987 Chem. Rev. 8729

[28] Brivio G P and Grimley T B 1993 Surf. Sci. Rep. 17 1-84

[29] Zhou Y, Pollak E and Miret-Artes S 2014 J. Chem. Phys. 140024709

[30] Sipkens T A and Daun K J 2017 Int. J. Heat Mass Transfer 11154

[31] Fan G and Manson J R 2009 Phys. Rev. B 79045424

[32] Pollak E and Tatchen J 2009 Phys. Rev. B 80115404

[33] Logan R M and Stickney R E 1966 J. Chem. Phys. 44195

[34] Grimmelmann E K, Tully J C and Cardillo M J 1980 J. Chem. Phys. 721039

[35] Logan R M and Keck J C 1968 J. Chem. Phys. 49860

[36] Hayes W W and Manson J R 2012 Phys. Rev. Lett. 109063203

[37] Hayes W W and Manson J R 2014 Phys. Rev. B 89045406

[38] Hayes W W and Manson J R 2011 J. Phys.-Condes. Matter 23484003

[39] Tully J C 1990 J. Chem. Phys. 92680

[40] Yan T, Hase W L and Tully J C 2004 J. Chem. Phys. 1201031

[41] Armand G and Manson J R 1982 Surf. Sci. 119 L299

[42] Pollak E and Miret-Artes S 2009 J. Chem. Phys. 130194710

[43] Pollak E 2011 J. Phys. Chem. A 1157189

[44] Pollak E and Miret-Artés S 2010 Chem. Phys. 375337

[45] Martin-Gondre L, Alducin M, Bocan G A, Diez Muino R and Juaristi J I 2012 Phys. Rev. Lett. 108096101

[46] Rittmeyer S P, Bukas V J and Reuter K 2018 Advances in Physics-X 31381574 Unsp

[47] Adelman S A and Doll J D 1976 J. Chem. Phys. 642375

[48] Polanyi J C and Wolf R J 1985 J. Chem. Phys. 821555

[49] Adelman S A and Doll J D 1976 J. Chem. Phys. 642375

[50] TullyJ C 1980 J. Chem. Phys. 731975

[51] Wang M C and Uhlenbeck G E 1945 Rev. Mod. Phys. 17323

[52] Plimpton S 1995 J. Comput. Phys. 1171

[53] Foiles S M, Baskes M I and Daw M S 1986 Phys. Rev. B 337983

[54] Zhou X W et al 2001 Acta Mater. 494005

[55] Hippler H, Troe J and Wendelken J 1983 J. Chem. Phys. 786709

[56] Zhen S and Davies G J 1983 Phys. Stat. Sol. (a) 78595

[57] Girifalco L A, Hodak M and Lee R S 2000 Phys. Rev. B 6213104

[58] Rudyak VY and Krasnolutski S L 2001 Dokl. Phys. 46897

[59] Wong R Y M, Liu C, Wang J, Chao CY H and Li Z G 2012 J. Nanosci. Nanotechnol. 122311

[60] Kington G L and Aston J G 1951 JACS 731929

[61] Vidali G, Ihm G, Kim H-Y and Cole M W 1991 Surf. Sci. Rep. 12133

[62] Kleiman G G and Landman U 1976 Solid State Commun. 18819

[63] Chon H, Fisher R A, McCammon R D and Aston J G 1962 J. Chem. Phys. 361378

[64] Head-Gordon M, Tully J C, Rettner C T, Mullins C B and Auerbach D J 1991 J. Chem. Phys. 941516

[65] Léonard C, Brites V, Pham T T, To Q-D and Lauriat G 2013 Eur. Phys. J. B 86164

[66] Mullins C B, Rettner C T and Auerbach D J 1989 Chem. Phys. Lett. 163111

[67] Hayes W W and Manson J R 2015 Surf. Sci. 64172

[68] Weinberg W H and Merrill R P 1972 J. Chem. Phys. 562881 